\title{
Les croyances collectives : un concept indéterminé
}

collective beliefs : an indeterminate concept

\section{Pascal Sanchez}

\section{OpenEdition}

Journals

Édition électronique

URL : http://journals.openedition.org/ress/3805

DOI : $10.4000 /$ ress.3805

ISBN : 1663-4446

ISSN : 1663-4446

Éditeur

Librairie Droz

Édition imprimée

Date de publication : 15 mai 2017

Pagination : 171-190

ISSN : 0048-8046

Référence électronique

Pascal Sanchez, «Les croyances collectives : un concept indéterminé », Revue européenne des sciences sociales [En ligne], 55-1 | 2017, mis en ligne le 15 mai 2020, consulté le 19 janvier 2021. URL http://journals.openedition.org/ress/3805; DOI : https://doi.org/10.4000/ress.3805 


\title{
LES CROYANCES COLLECTIVES: UN CONCEPT INDÉTERMINÉ
}

PASCAL SANCHEZ

Université Lumière Lyon-II pascal.sanchez@gmx.fr

Résumé. Les croyances collectives font figure de question classique de la sociologie et de l'anthropologie. Pourtant la notion de «croyances collectives» demeure encore largement indéterminée en dépit des nombreuses contributions théoriques imaginées en vue de les analyser. Pour comprendre ce paradoxe, il faut revenir au concept lui-même, le décomposer en ses multiples sens possibles afin de montrer les limites de l'attitude réductionniste qui prévaut encore aujourd'hui en sociologie et en anthropologie.

Mots-clés: acceptations collectives, croyances collectives, modalités de la croyance, représentations partagées, théories sociologiques et anthropologiques.

\begin{abstract}
Collective beliefs appear to be a classical topic of sociology and anthropology. Nevertheless the notion of "collective beliefs" still remains widely indeterminate in spite of the multiple theories produced to analyze them. To understand this paradox, it is necessary to return to the concept itself, to break it down in its multiple possible meanings in order to highlight the limits of the reductionist attitude which still prevails today within sociology and anthropology.
\end{abstract}

Keywords: belief modalities, collective acceptances, collective beliefs, shared representations, sociological and anthropological theories. 


\section{INTRODUCTION}

Depuis plus de cent ans, sociologues et anthropologues se sont affrontés sur le terrain de l'explication des croyances collectives. L'enjeu épistémologique est, à l'évidence, décisif: rendre compte des croyances collectives c'est démontrer du même coup la capacité de ces sciences à expliquer ce qui apparaît comme étant, au premier abord, énigmatique, voire irrationnel. Pourtant, et c'est là un paradoxe saisissant, malgré les nombreux travaux consacrés à cette question qui comptent parmi les plus belles réussites de la sociologie et de l'anthropologie, le statut des croyances collectives est toujours en débat et semble largement indéterminé. Les limites rencontrées par les principales écoles en matière d'explication des croyances collectives illustrent, par-delà leurs différences et oppositions, les difficultés éprouvées par la sociologie et l'anthropologie à saisir cet objet. Ce paradoxe troublant - application successive de différents filtres théoriques à partir des insuffisances du modèle précédent, immensité de la littérature d'une part, et perpétuels débats sur l'explication des croyances collectives d'autre part - tient à l'absence de problématisation de la notion elle-même, notion particulièrement complexe en ce qu'elle recouvre de multiples sens qui ne se réduisent pas à une seule expression.

Cet article vise à construire le concept de «croyances collectives» pour en déduire des préconisations méthodologiques. Il tente ainsi d'éclaircir notre paradoxe et milite plus fondamentalement sur la nécessité de décomposer le concept de «croyances collectives » en ses diverses dimensions afin de comprendre à quoi se réfèrent la sociologie et l’anthropologie lorsqu'elles convoquent cette notion.

\section{L'INDÉTERMINATION DES CROYANCES COLLECTIVES EN SOCIOLOGIE ET ANTHROPOLOGIE}

L'indétermination des croyances collectives en sociologie et en anthropologie peut être établie en parcourant la production d'auteurs représentatifs des principaux courants théoriques recensés (évolutionnisme, fonctionnalisme, structuralisme, individualisme méthodologique, naturalisme). L'histoire de la sociologie et de l'anthropologie est, à l'évidence, bien plus complexe que 
cette représentation commode découpant en tranches épaisses l'ordre successif des diverses expressions théoriques. Chaque courant enregistre, en effet, en son propre sein des écarts, voire des oppositions fortes. Andrew Lang (I90I) a ainsi vertement critiqué James G. Frazer et le fonctionnalisme de Bronislav Malinowski n'est pas identique à celui d'Alfred Radcliffe-Brown. De même, le point de vue d'un auteur, sur un thème identique, peut révéler des inflexions dans le temps, comme ce fut le cas notamment pour Émile Durkheim (I899, 1985 [1912]) et pour Frazer (Ackerman,1987). Cette histoire, entreprise ailleurs dans ses multiples ramifications à partir du concept de magie (Sanchez, 2007), restituée ici quà grands traits à travers quelques figures consacrées, signale l'extrême difficulté rencontrée par l'anthropologie et la sociologie à se saisir de la notion de croyances collectives.

L'évolutionnisme a permis l'émergence du concept de croyances collectives en tant qu’objet digne d’analyse et d'interprétation. L'importance historique de l'évolutionnisme est d’avoir installé les croyances magiques et religieuses dans le champ des représentations collectives, et ce dès les premiers écrits d’Edward B. Tylor (1876 [1871]) et de William R. Smith (I889). Cependant, par un étrange paradoxe, au moment même où l'évolutionnisme jetait les bases de l'analyse des croyances collectives, il engageait une des formes les plus radicales de leur disqualification. Chez Tylor, comme chez Frazer (1993 [1906-1915]), la religion et la magie primitives ne tiennent qu’à un fil ténu, celui de l’application de quelques principes associatifs de la pensée qui relient indûment des événements entre eux sur la base de cette confusion propre aux primitifs : la confusion entre association causale et association idéale. Sans doute, l'évolutionnisme a-t-il soulevé une question essentielle - comment expliquer rationnellement des croyances apparemment absurdes - mais en des termes qui obscurcissaient grandement le concept de croyances collectives dans la mesure où le voile d'illusion cognitive dans lequel les primitifs sont censés être plongés suffit à rendre compte du phénomène.

Réagissant à l'évolutionnisme, le fonctionnalisme a affirmé la dimension rationnelle des croyances à partir de postulats méthodologiques bien mis en exergue par Radcliffe-Brown (1972 [1952]) et Robert K. Merton (1968 [1949]). Une croyance est rationnelle en ce qu'elle s'insère dans les rouages de la reproduction d'un système tendant à demeurer stable (Malinowski, 1992 [1948]) ou, 
dans une autre variante, en ce qu'elle contribue à la dynamique des systèmes (Marwick, 1952, Turner, 1957). En partant de la distinction implicite entre l'idéel et le réel et en faisant des représentations un simple décalque des enjeux sociaux, le fonctionnalisme a voulu dissoudre les croyances dans le collectif en réduisant celles-ci à une fonction d'utilité pour le groupe. De fait, si le fonctionnalisme est souvent parvenu à indiquer par quels mécanismes une croyance aussi stupéfiante que la divination peut s’avérer utile (Moore, 1959), il a échoué tout aussi régulièrement à faire de cette fonction d'utilité la source d’une croyance possible (Adler et Zempleni, 1973).

Le structuralisme, à travers son principal promoteur, a préféré contourner ce concept. Claude Lévi-Strauss (1989 [1950]) nous enjoint en effet de dépasser le plan phénoménologique, en particulier de dépasser l'ordre des sentiments et des croyances qui, au regard d'une explication sociologique, ne constitue pas un objet, soit parce que cet ordre n’est qu’un épiphénomène, soit parce qu'il demeurera mystérieux tant qu'un travail d’abstraction ne sera pas entrepris indépendamment des points de vue portés par les croyants eux-mêmes. C'est ainsi que Lévi-Strauss reproche fortement à Marcel Mauss de s'être servi de représentations indigènes (hau, mana) pour conceptualiser les dimensions de l'échange. L’anthropologue doit, bien au contraire, reconstruire les modalités de la croyance dans le langage de l'efficacité symbolique qui ne doit rien, ou si peu, à l'expérience vécue (Lévi-Strauss, 1985 [1958]). Le structuralisme, dans son expression lévi-straussienne, conduit ainsi à une forte relativisation de la thématique des croyances collectives en ce qu'il surévalue la dimension symbolique au détriment d'autres questions, en particulier la dimension pratique (Bourdieu, 1984).

L'individualisme méthodologique, tel qu'il a été incarné en France par Raymond Boudon (1986, I990), présente l'incontestable mérite de mettre la question des croyances au cœur de toute l'entreprise sociologique. Croire, c'est se référer à un système argumentatif souple et ouvert qui explicite les raisons, relatives et limitées à un contexte, par lesquelles le croyant adhère légitimement à des représentations perçues par lui-même comme fondées et rationnelles et à travers lesquelles son action devient compréhensible du point de vue d'un observateur extérieur. Boudon (1998) rapproche et refor- 
mule de la sorte les positions de Durkheim et de Weber, habituellement présentées comme opposées, en matière d'analyse des croyances collectives. L'individualisme méthodologique n’est cependant pas parvenu lui aussi à problématiser la notion de «croyances collectives », tant du côté des croyances que du côté du collectif: du côté de la croyance en ce qu'il déploie toujours la même mécanique explicative en présupposant ainsi que la thématique de l'adhésion constituerait le modèle unique d'intellection de toutes les formes de croyance (Clément, 1999); du côté du collectif en ce qu’en réduisant la question de la croyance à l'usage d'une capacité cognitive, l'individualisme méthodologique n'est plus en mesure d'établir cette différence fondamentale entre, d'une part, croire en une proposition et, d'autre part, croire en une représentation collective, et ce dans toutes les dimensions cognitives, sociologiques, symboliques et politiques que cette dernière notion recouvre.

Lapproche naturaliste, infra-individualiste (Sperber, 1996), aborde les croyances collectives non sous l'angle de leur production et adhésion, mais sous celui de leur distribution et diffusion. Elle tend à faire disparaître du débat la question vectorielle qui a dominé toute l'anthropologie et la sociologie des croyances. Les diverses interprétations peuvent se résumer à une alternative en forme d'aporie pour le naturalisme: le vecteur de la croyance doit-il partir du croyant, de ses raisons et de sa position, pour aller vers les croyances, ou bien doit-il partir des croyances elles-mêmes, de leurs conditions sociales de production, pour aller vers les croyants? La naturalisation des croyances collectives pose autrement la question; certaines propositions religieuses, au sens large du terme, ont la faculté de se répandre et de devenir publiques, non en raison de leur signification pour le sujet ou de leur utilité pour le groupe, mais parce qu’elles répondent adéquatement aux capacités d'une infrastructure matérielle qui s'est structurée au cours de l'évolution: notre cerveau. En somme, le salut de l'anthropologie et de la sociologie des croyances collectives repose sur l'espérance d'une compréhension achevée des capacités de notre équipement neuronal à produire des représentations. 
L'approche naturaliste n'est à ce jour qu'un pari, un pari qui renferme de surcroît des hypothèses réalistes et atomistes en matière de représentations mentales difficilement acceptables (Lenclud, 1998). Plus fondamentalement, cette approche n'est pas en mesure de rendre compte des particularités des croyances collectives. Toute croyance doit certes comporter des caractéristiques mémorisables et diffusables, mais toute représentation devenue publique n’a pas pour autant le statut de croyance. Il faut donc autre chose à une représentation mentale pour devenir une croyance (Bronner, 20ı) et cette différence échappe encore à l'entreprise naturaliste. Le collectif ne peut être non plus ramené, dans le cadre d'une analyse des représentations, à un espace neutre simplement voué à favoriser les échanges et la transmission de messages. En effet, une telle approche relativise toute entreprise de contextualisation sociale et historique et n'est plus dès lors en mesure de répondre à la question touchant, par exemple, à la distribution dans le temps et dans l'espace de certaines représentations relatives aux sorcières dans l’Europe médiévale et de la Renaissance. Pour répondre à une telle question (Macfarlane, 1970; Thomas, 1972), décomposer la croyance en différents atomes de propositions pour en établir leur aptitude à se diffuser ne suffirait pas. Il faudrait, de surcroît, pouvoir restituer la cohérence d'un système de représentations, décrire les mécanismes d’accusation dans le cadre étroit de communautés villageoises tout en intégrant dans l'analyse cet effet de contingence historique lié à la centralisation du pouvoir. Une analyse des croyances collectives ne peut être ainsi réduite à une épidémiologie des représentations dans la mesure où une telle analyse doit nécessairement mêler différents plans : le cognitif, le social et l'historique.

L’insuffisante problématisation de la notion de croyance collective, telle qu'il est possible de la restituer à travers quelques figures majeures de la sociologie et de l'anthropologie, renvoie à une constante hésitation entre Charybde et Scylla : faut-il, dans l'explication des croyances collectives, surestimer le poids des croyances elles-mêmes, leur dynamique propre et les mécanismes d’adhésion, au risque de sous-estimer par-là même le collectif, à savoir les logiques qui commandent l'unité et la reproduction du groupe, ou bien faut-il sous-estimer les croyances au risque de surestimer le collectif? Croyons-nous parce que c'est collectif ou bien 
produisons-nous du collectif parce que nous croyons, telle est la principale antinomie qui a traversé de part en part la thématique des croyances collectives.

Cette antinomie, sous forme de «ou bien ou bien», signale la difficulté propre à l'ambition explicative de la sociologie et de l'anthropologie. Cette difficulté porte sur la nécessité de problématiser, et non de séparer ou de distinguer, trois dimensions à la fois : la croyance, le collectif qui sous-tend la croyance, et la liaison entre la croyance et le collectif - liaison qui apporte précisément une dimension supplémentaire au problème et qui fait de la notion de «croyances collectives » un objet bien spécifique. Cette liaison est l'enjeu même de la sociologie et de l'anthropologie des croyances collectives. Par-delà leur opposition, les principales théories sociologiques et anthropologiques ne sont pas véritablement parvenues à construire une telle liaison et ont partagé la même difficulté à restituer ce que l'expression «croyances collectives » veut dire, et ce pour de nombreuses raisons. Elles ont cherché à établir une proposition - l'homme fait selon ce qu'il croit ou croit selon ce qu'il fait - en faisant toutefois l'économie d'une analytique de la croyance non seulement en tant que contenu mais aussi en tant qu'expérience. Elles ont sous-estimé, dans l'ensemble, toutes les nuances rattachées au fait de croire et se sont focalisées principalement sur la croyance en ce qu'elle permet de produire comme ordre social et symbolique, en ce qu'elle donne accès à une compréhension d'un événement ou d'un phénomène, en ce qu'elle se conforme à des règles de transmission des représentations. Elles ont établi, par conséquent, une sorte de correspondance parfaite entre croire et agir dans la mesure où l’action, commandée par des rites ou bien reliée à un système argumentatif, est la seule instance capable de matérialiser et de dévoiler une représentation collective fondamentalement inobservable (Sperber, 1974).

En définitive, l'image des croyances collectives renvoyée par ces diverses théories apparaît comme une tentative de schématisation d'une question redoutablement complexe, une question qui ne se laisse pas enfermée dans les diverses propositions unilatérales suivantes adoptées au cours de l'histoire : croire c'est associer des idées; croire c'est révéler un ordre social; croire c'est agir et penser selon les modalités de l'efficacité symbolique ; croire c'est argumenter; croire c'est partager des représentations mentales particulières. 
De beaux travaux ont bien évidemment ponctué cette histoire, ceux, à titre d'exemples, d'Edward E. Evans-Pritchard (1976 [1937] et de Jeanne Favret-Saada (1994 [1977]), en ce qu'ils ont précisément réussi à relier, en un tableau suggestif, les différentes dimensions d'une croyance collective en l'élevant au rang d'une expérience vivante. Par-delà les nombreuses et incontestables réussites, le point dominant de cette histoire c'est la constante difficulté éprouvée par la sociologie et l'anthropologie à fournir des explications convaincantes sur cette fascinante faculté humaine à produire et à adhérer à des croyances collectives, souvent marquées du sceau de l'irrationalité apparente. Deux stratégies dévoilent particulièrement bien les difficultés rencontrées par la sociologie et l'anthropologie pour rendre compte des croyances collectives, lesquelles expriment une sorte de méfiance à l'égard d'une notion devenue, avec le temps, délicate à manipuler (Hamayon, 2005).

La première stratégie consiste à extraire du discours anthropologique et sociologique tout un impensé interdisant l’accès à une juste représentation du concept de «croyances collectives». Il convient en particulier de se défaire de cette relation, faussement universelle, issue de notre tradition associant croyance et représentation. Pour cela, il suffit de partir de la langue vernaculaire des croyants, des catégories de la pensée qu'elle charrie, pour être en mesure de restituer les diverses modalités possibles de la croyance (Pouillon, 1979; Lenclud, 1990; Needham, 1972). Il faut enfin penser les croyances collectives à partir des questions qu'une culture se pose à elle-même, les problèmes majeurs qu'elle entend résoudre à travers les représentations qu'elle sécrète, et non plaquer des interrogations qui lui sont profondément étrangères, en particulier les mécanismes cognitifs établissant les conditions d'une correspondance entre la croyance et la réalité (Winch, 1986).

Ces injonctions méthodologiques sont sans doute utiles en ce qu'elles préservent la sociologie et l'anthropologie du piège du réalisme conceptuel (Hayek, 1952). Elles confortent cette salutaire vigilance épistémologique qui conduit opportunément à réinterroger toutes nos évidences rattachées à la notion de croyance collective (Piette, I999; Sheid, 2005). Cependant, ces mises en garde méthodologiques comportent deux limites. En premier lieu, bâtir une 
anthropologie et une sociologie du point de vue des catégories de l'autre est un projet désespéré car il implique, pour être véritablement accompli, l'obligation de se défaire de tout l'héritage conceptuel par lequel des questions et des objets adviennent. La radicalité du «tout reprendre à zéro» (Jorion, I986) n’est d'ailleurs pas une nécessité. Les travaux d’Evans-Pritchard (I954) ont abondamment démontré qu'il est possible de restituer les particularités langagières de la pensée nuer sans pour autant renoncer au principe de raison, c'est-à-dire aux principes de cohérence des représentations et de la confrontation, selon des dispositifs propres à chaque culture, de ces représentations à la réalité. Les approches qui rattachent la croyance à des découpages langagiers présentent enfin l'inconvénient majeur de surestimer amplement l'importance du langage dans la construction des représentations (Rosch, I980).

La seconde stratégie relie l'effort de construction du concept de croyances collectives à une démarche typologique. Il s'agit non seulement de classer les croyances collectives pour fixer des repères dans cet univers foisonnant mais aussi, par ce geste, de proposer une clé d’interprétation. Différentes typologies ont été proposées : celle de Gérald Bronner (2003) qui renvoie au degré de systématisation de l'argumentation et au contenu de vérité des propositions d'une croyance; celle de Boudon (2008) qui repose sur les formes de confrontation avec la réalité ; celle, complémentaire et prenant appui sur les précédentes, que nous avons nous-même proposée (Sanchez, 20o9). Ces typologies ont pour principal intérêt de circonscrire l'horizon du questionnement, le lieu à partir duquel il est possible de penser les croyances collectives. Pour ces typologies, analyser des croyances collectives revient au fond à interroger leur degré de rationalité. Du coup, elles opèrent subrepticement un déplacement de la problématique en postulant que la croyance se meut nécessairement dans l'horizon du rationnel. Plus fondamentalement, ces approches typologiques ne pénètrent pas au cour de la question en ce qu'elles ne disent pas, par-delà les différentes formes repérées et classées, ce que le collectif ajoute au concept de croyance ni ce que la croyance ajoute au concept de collectif. Les différentes formes sont de fait déjà données et désignées (religion, magie, superstition, etc.) et il est question de les différencier à l’intérieur d'un espace précédemment circonscrit. 


\section{2. ÉLÉMENTS D'UNE CONSTRUCTION DU CONCEPT DE CROYANCES COLLECTIVES}

Par-delà leurs différences, un point commun rassemble les diverses théories anthropologiques ou sociologiques. Quelle que soit leur orientation fondamentale, elles déploient leurs points de vue et perspectives en l'absence d'un travail approfondi et systématique d'éclaircissement du concept de «croyances collectives», comme si cette notion relevait de ces évidences qui rendraient vaine toute démarche réflexive. Une fois la notion de «croyances collectives» posée à titre de postulat, arrimée à une définition implicite, les théories sociologiques et anthropologiques n'auraient plus qu'à suivre l'inclinaison de leur pente doctrinale pour dérouler leurs diverses positions. L'évaluation critique des contributions de la sociologie et de l'anthropologie convie ainsi à orienter le regard en-deçà des théories elles-mêmes, au niveau du concept, afin de comprendre les raisons de leur défaillance.

Sur cette question, il est non seulement utile mais aussi incontournable de prendre appui sur la philosophie qui a apporté une contribution décisive à la construction de ce concept. La croyance est un concept issu de la tradition philosophique (Ricœur, 1968), concept qui a été traditionnellement pensé de manière résiduelle, à partir d'un écart entre croire et connaître, et qui a été plus récemment compris comme une attitude propositionnelle (Russell, 200I [19I2]). Pour une grande part, la sociologie et l'anthropologie sont les héritières de cette problématisation philosophique en ce qu'elles ont principalement évalué les croyances collectives en fonction de leur distance, plus ou moins marquée selon les approches, au rationnel et en fonction du critère fondamental de confrontation au réel d'un ensemble de propositions constitutives d'une croyance. Cependant, sociologues et anthropologues ont été, dans l'ensemble, peu intéressés par tout un travail analytique engagé par certains philosophes désireux de construire, avec le plus de concision possible, le concept de croyance.

Dans ce cadre d'élucidation conceptuelle, la croyance a été nettement distinguée de l'acceptation (Cohen, I992; Tuomela, 2000). Croire c'est autre chose qu'accepter volontairement une proposition en raison d'un intérêt pratique, c'est, à l'inverse, entretenir une relation réflexive ou tacite à des propositions 
complexes accompagnées d'attitudes correspondantes et guidées par la norme du vrai (Engel, 1997). Poursuivons encore: il convient, plus précisément, de ne pas confondre croire avec juger, accepter ou admettre. On peut croire sans porter un jugement, en tant que disposition, mais on peut aussi croire, de façon réflexive, en validant, à des degrés variables, l'élément de vérité de la croyance. On peut, de même, accepter une proposition sans y croire, par intérêt ou parce que le contexte y convie, et l'on aussi peut admettre une proposition sans y croire et sans adopter le comportement correspondant.

Ces distinctions ne vont pas de soi et demandent à être approfondies. Elles ont fait l'objet de nombreux débats. Sur la base de ces distinctions, les croyances collectives peuvent être en effet réduites à des formes de représentations partagées ou d'acceptations collectives entre les membres d’un groupe (Meijers, 1999), conception reposant sur une approche agrégative de la production des croyances collectives qui laisse de côté tout le processus fondamental d'engagement mutuel (Gilbert, 2002). En opérant ces premières distinctions, la philosophie accomplit sa fonction primordiale, celle d'introduire du doute et de la complexité autour d'une notion qui semblait auparavant relever de l'évidence. Fabrice Clément (20I0) s'inscrit dans cette lignée. Il identifie ainsi six manières d'éprouver, à travers des comportements et des évaluations, des croyances. Parmi ces six façons de produire de la croyance, quatre peuvent être considérées comme des croyances collectives, soit parce qu'elles ont été fournies par le groupe et ne font pas ou peu l'objet d’une évaluation réflexive de la part de l'individu (schémas sociaux, croyances endossées), soit parce qu'elles renvoient à des processus, d’intensité variable, d’adhésion ou d’acceptation de propositions par l'individu (croyances semi-propositionnelles, croyances délibératives). Il faut dès lors renoncer à subsumer le divers du phénomène sous un unique concept de «croyances collectives ». Ce renoncement délivre en fait un message positif car œecuménique : des approches théoriques, profondément différentes dans leurs orientations, peuvent cohabiter sans se réfuter. En s'attaquant préférentiellement à l'une des formes revêtues par les croyances collectives, les théories sociologiques et anthropologiques espèrent, à tort, atteindre une essence là où il n'y a que des manifestations diverses de la croyance commandant le choix d'outils méthodologiques adaptés. 
Cette approche stratifiée constitue une indéniable avancée en clarifiant grandement les différents sens possibles attribués aux croyances collectives. Elle donne, dans la foulée, des indications intéressantes sur la voie à suivre en matière de construction du concept de «croyances collectives », mais cet effort doit être poursuivi et amplifié en décomposant les deux termes (croyance, collectif) du problème et en indiquant plus précisément leur articulation.

En premier lieu, la notion de croyance doit être restituée à l'intérieur d'un continuum d'attitudes adoptées à l'égard d'une proposition ou d'un événement, attitudes évolutives se transformant en fonction des circonstances et diversement adoptées et vécues par les membres d'un groupe. Croire regroupe ainsi des situations et des expressions différentes, de surcroît très difficiles à repérer et à qualifier dans la mesure où les croyances ne constituent pas un domaine séparé mais imprègnent fortement les pratiques, les représentations du temps et de l'espace, les manières d'être et les rapports aux autres au point qu'il est mal aisé de démêler l'écheveau et de circonscrire un territoire qui serait propre aux croyances. Ces modalités de la croyance couvrent un large spectre de positions se combinant dans des proportions variables en fonction des individus, des groupes et des contextes. Croire peut se dire de six façons différentes décrivant une échelle d'intensité, chacune de ces six façons intégrant par ailleurs des nuances d'expression. L'intensité la plus faible et qui exprime une position négative à l'égard d'une croyance passe par l'expression d'un rejet implicite ou ouvertement critique. Ce rejet résulte soit d'une acceptation antérieure, et il s'agit alors d'un abandon, soit d'une résistance ou d'un refus revendiqués. Croire peut ensuite adopter la forme du doute, expression intime ou publique, doute qui peut renvoyer à une attitude générale sceptique ou à une attitude inquiète conduisant à vouloir établir la véracité de la croyance par sa mise à l'épreuve et au moyen de la validation des principales propositions. Croire c'est encore accepter un ensemble de propositions pour des raisons externes à celles-ci, soit parce qu'elles sont incarnées par des acteurs ou des institutions considérés comme légitimes et investis d'une certaine autorité du point de vue de ceux qui les acceptent, soit par transmission, tradition ou par habitude. Croire c'est, quatrièmement, admettre des propositions comme un prérequis fournissant des règles de compor- 
tement et de coordination de l'action collective, des repères de représentation, soit à titre d'hypothèse implicite, soit dans le cadre d'un processus d'échanges et de partage de propositions. Croire c'est, cinquièmement, adhérer à des propositions, soit en raison de leur puissance argumentative et de leur degré de correspondance à la réalité, soit en raison de leur capacité à délivrer du sens et à interpréter la réalité. Croire ce peut être enfin éprouver en plus d’approuver. Croire devient ici, dans son intensité la plus forte, une condition d'existence, la clé de voûte à laquelle toutes les représentations et actions se rattachent et sont subordonnées, intensité forte obtenue soit sous forme d'expérimentation, soit sous forme d'identification à une idéologie au sens large du terme. Cette dernière modalité de la croyance est fondamentale car elle ne se réduit pas à la seule dimension d'une attitude propositionnelle. Croire exprime ici le rapport au monde propre à un individu ou à un groupe, une manière de combiner des expériences à partir d'une recherche antéprédicative de sens qui n'est pas assujettie à un exercice d'évaluation du degré de vérité des propositions d'une croyance. Il suffit, pour s'en convaincre, de se reporter aux mécanismes d'adhésion à des croyances fortement engageantes, celles par lesquelles un individu affirme ce qu'il est à travers ce à quoi il croit (Huguenin, 1995; Sauvayre, 2012).

Dans l'examen des croyances collectives, il convient de prendre toute la mesure de la diversité des modalités de la croyance en renonçant à élever l'une d'entre elles au rang d'une expression générale. Ce renoncement met non seulement fin à cette orientation réductrice qui a souvent traversé les théories successives, orientation commune qui explique en grande partie leur échec, mais fait aussi surgir de multiples et passionnantes questions. Parmi ces questions: comment s'expriment ces diverses modalités dans le temps, selon les sociétés ou les groupes? Pourquoi telle modalité a-t-elle tendance à devenir dominante dans le temps, dans une société ou un dans groupe ? Comment s'opère le passage d'une modalité à une autre dans le temps, dans une société ou dans un groupe? Quelles sont les représentations et les pratiques rattachées aux différentes modalités dans le temps, dans une société ou dans un groupe? Quelle est la place donnée à diverses modalités de la croyance selon les époques, les sociétés ou les groupes? Ces questions ont fait l'objet de quelques beaux travaux, à l'exemple de ceux de Lucien Febvre sur les 
formes de l'incroyance adoptées à la Renaissance (Febvre, 1968), mais n'ont pas véritablement constitué un programme de recherches.

Ces questions opèrent un véritable déplacement du regard en ce que la diversité de la croyance ne devient plus le problème à éliminer mais bien l'interrogation première. L’analyse des croyances collectives doit ainsi débuter par l'élaboration d'un tableau complet et descriptif des diverses formes adoptées ou rejetées dans un contexte. En procédant de la sorte, il s'agit d'expliciter que veut dire croire pour un groupe et les raisons pour lesquelles il tend à privilégier une forme, à associer différentes formes entre elles ou à en éliminer d'autres.

Reste l'autre face du concept: il s'agit de comprendre en quoi le «collectif» apporte une dimension particulière et supplémentaire à l'expression des modalités du croire. La notion de «collectif» désigne fondamentalement ce qu'est la double vocation des groupes - faire et être ensemble -, le défi étant de comprendre comment l'action et la représentation deviennent possibles au sein des groupes. Le collectif est de fait tout autant «stratifié» que la croyance. Le collectif, au plus bas de l'échelle de structuration, peut désigner la série, le regroupement nominal, sous une même étiquette, d'individus, faiblement interdépendants, et non reliés entre eux par la conscience et la volonté de faire groupe. La file d'attente d'un bus, pour reprendre cette image archétypale (Sartre, 1960), illustre ce qu'est une série. Il peut prendre la forme d'une agrégation d'individus au sens où les individus ont conscience de leur proximité d'intérêts ou de représentations, de leurs interdépendances, sans pour autant vouloir former un groupe doté de représentations communes et capable de coordonner l'action, paradoxe qui a donné lieu à une immense littérature (Olson, 1978 [1966]). L'association est une autre image du collectif. Il s'agit, dans ce cas de figure, d'individus conscients de leurs intérêts et de leurs interdépendances, partageant des représentations communes et désireux d’accomplir ensemble un projet connu de tous ou d'éprouver à plusieurs une expérience similaire. Le collectif résulte à la base de choix individuels mais l'association crée une nouvelle réalité, un «sujet pluriel» exerçant un effet de contrainte dans les actes qui semblent même les plus libres en apparence (Gilbert, 1989). L'organisation, au plus haut point de l'échelle de structuration, désigne des indi- 
vidus intégrés à un groupe dont la fonction est de produire des représentations communes et de coordonner l'action collective au nom et à la place de tous ses membres, et ce le plus souvent dans le cadre d'une institution.

Cette présentation à grands traits des formes du collectif, dont chacune des expressions renvoie à des expériences très différenciées d'être et d'agir ensemble, introduit là encore un doute quant à la possibilité d'atteindre un sens général relatif à la notion de «croyances collectives». Il y a tout de lieu de penser que ces formes affectent différemment les modalités du croire, qu’elles ne sont pas neutres quant à la manifestation de la croyance. La difficulté, en matière de croyances collectives, consiste dès lors à repérer les types, souvent entremêlés, de collectif présents dans un groupe et à saisir leurs logiques de fonctionnement et de transformation, la réalité, bien évidemment plus composite, ne se laissant pas emprisonner dans les filets aussi lâches que ceux exposés plus haut. La prise en compte de ces multiples figures du collectif fait ainsi surgir de nouvelles questions essentielles à la compréhension des croyances collectives. En particulier: une croyance collective est-elle ou non associée à une forme préférentielle du collectif et ce pour quelles raisons? Les diverses modalités du croire sont-elles ou non reliées à une ou à des formes du collectif et ce pour quelles raisons? Le passage d'une figure du collectif à une autre se traduit-il ou non par une modification de contenu et d'expression d'une croyance et ce pour quelles raisons? Expliquer une croyance collective c'est donc restituer ce que croire veut dire dans le cadre d'un groupe dont il convient de déterminer les principes de structuration, c'est-à-dire la façon dont celui-ci parvient à créer du collectif, dans la mesure où les formes du collectif exercent une influence sur les modalités du croire.

La problématique du collectif adossé aux croyances ne se résume pas à la seule interrogation des principes de structuration des groupes et de leurs interrelations avec les modalités du croire. À cette question relative à la forme s'ajoute une autre, tout aussi essentielle, concernant la signification du collectif même. Le collectif peut adopter trois expressions différentes ajoutant un degré supplémentaire de complexité dans l'analyse du concept. Par collectif, il est possible d'entendre ce qui est commun à plusieurs individus en termes de représentation, sans pour autant que ce commun conduise à des modalités semblables de croire 
ou à une argumentation et à des pratiques similaires. C'est à cette représentation du collectif que se réfèrent les adeptes de la conception sommative des croyances collectives : il y a formation d'une croyance collective si tous les individus d'un groupe croient p. Le collectif désigne, en un second sens, un partage de représentations au sein d'un groupe, l'argumentation et l'échange étant au cœur de l'élaboration d'une croyance collective. Le partage des représentations ajoute une nouvelle condition à la conception sommative qui est celle d'un savoir commun, a minima implicite, le plus souvent rendu explicite (Bratman, 1999). Le partage de représentations ne se traduit pas nécessairement par l'alignement des modalités de croire et des pratiques au sein du groupe car ce qui est mis ici en commun c'est seulement la croyance elle-même, son contenu. Le collectif, au sens fort, adjoint à ces deux premières dimensions du commun et du partage une clause additionnelle d'unité et de convergence relative aux modalités de croire et aux pratiques corrélativement liées à la croyance. Au sens fort, les croyances collectives désignent l'existence des représentations communes parmi les membres d'un groupe, des représentations partagées et circulant entre les membres, des modalités de croire semblables accompagnées de pratiques associant des signifiants à un même signifié. C'est cette conception-là des croyances collectives que Durkheim (2010 [1898]) avait en tête lorsqu'il tenta d'en démontrer l'existence.

Ces trois significations du collectif ne dessinent pas des univers stables et étanches; selon les contextes, les individus, les propositions et les expériences, une croyance peut successivement revêtir ces trois visages. Elle peut, de même, faire cohabiter en elle, dans des proportions variables, toujours selon les individus et les contextes, ces trois manières d'exprimer le collectif. Evans-Pritchard (1976 [1937]) l'avait au fond déjà largement suggéré en exhibant les vingt-deux mécanismes inventés par les Azandé pour assoir la pertinence de leurs croyances, mécanismes qui relèvent à la fois du commun, du partage et du collectif au sens fort du terme. Dans la même veine, Hagenbucher Sacripanti (1992) montre comment des représentations traditionnelles rattachées au sida passent d'un registre à un autre, du registre du collectif au sens fort à celui des représentations partagées. 


\section{CONCLUSION}

La notion de «croyances collectives » peut, en définitive, être restituée à travers 72 combinaisons possibles. Il suffit pour cela de croiser les trois axes (six modalités du croire, quatre formes et trois sens du collectif) par lesquels cette notion a été démembrée pour être en mesure de délimiter l'étendue de son espace théorique. Sans doute, ce chiffre s'apparente à un exercice de l'esprit en ce qu'il est peu probable de rencontrer dans la réalité de pareilles combinaisons. Cet exercice montre cependant l'intérêt pratique qu'il y a à construire le concept de «croyances collectives » préalablement à tout travail empirique. La compréhension d'une croyance collective requiert que nous identifiions la combinaison sous laquelle elle se manifeste et les raisons pour lesquelles elle s'offre à l'analyse empirique sous cette forme.

Cette décomposition du concept indique de même toutes les limites du réductionnisme auquel souvent cèdent les sociologues et les anthropologues en suivant les injonctions propres à certaines écoles. Ils auront tendance à se porter vers la combinaison qui sera la plus conforme à leurs options méthodologiques pour penser les croyances collectives, à se tourner vers certaines croyances ou vers certaines caractéristiques des croyances pour déployer leurs analyses en terrain conquis.

Les croyances collectives est un objet possible des sciences sociales. La relative indétermination du concept n’est pas un argument décisif militant pour la dissolution du problème. Construire ce concept, c'est prendre acte de la diversité du phénomène mais aussi de la profondeur des questions charriées par les croyances collectives rendant illusoire l'atteinte d’une formulation générale et unique.

\section{BIBLIOGRAPHIE}

ACKERMAN R., 1987, J.G. Frazer, his Life and Work, Cambridge, Cambridge University Press.

ADLER A. et ZEMPLENI A., 1972, Le Bâton de l'aveugle. Divination, maladie et pouvoir chez les Moundang du Tchad, Paris, Hermann.

BOUDON R., 1986, L'/déologie ou l'origine des idées reçues, Paris, Fayard.

-, 1990, L'Art de se persuader des idées fragiles, douteuses ou fausses, Paris, Fayard. 
-, 1998, Études sur les sociologues classiques, I, Paris, PUF.

-, 2008, Le Relativisme, Paris, PUF.

BOURDIEU P., 1984, Le Sens pratique, Paris, Éditions de Minuit.

BRATMAN M., 1999, Faces of Intention, Cambridge, Cambridge University Press.

BRONNER G., 2003, L'Empire des croyances, Paris, PUF.

-, 2010, «Le succès d'une croyance. Évocation-crédibilité-mémorisation », L'Année sociologique, 60-I, p. 137-160.

CLÉMENT F., 1999, «la sociologie cognitive: une bien étrange croyance», Cahiers internationaux de sociologie, CVII, p. 89-104.

-, 2010, «La nature des croyances collectives», L'Année sociologique, 60-I, p. 63-91.

COHEN L.J., 1992, An Essay on Belief and Acceptance, Oxford, Clarendon Press.

DURKHEIM É., 1899, «De la définition des phénomènes religieux», L'Année Sociologique, II, p. I-28.

-, 2010 (1898), «Représentations individuelles et représentations collectives», in Id., Sociologie et Philosophie (1924), Paris, PUF, p. I-48.

-, 1985 (1912), Les Formes élémentaires de la vie religieuse, Paris, PUF.

ENGEL P., 1997, «Croyances collectives et acceptations collectives», in R. Boudon,

A. Bouvier et Fr. Chazel (dir.), Cognition et sciences sociales, Paris, PUF, p. I55-I73.

EVANS-PRITCHARD E.E., 1976 (1937), Witchcraft, Oracles and Magic Among the Azande, Oxford, Clarendon Press.

-, 1954, «A Problem of Nuer Religious Thought», Sociologus, 4- I, p. 23-4I.

FAVRET-SAADA J., 1994 (1977), Les Mots, la mort, les sorts, Paris, Gallimard.

FEBVRE L., 1968 (1942), Le Problème de l'incroyance au Xve siècle, Paris, Albin Michel.

FRAZER J.G., 1993 (1906-1915), Le Rameau d'or, Paris, Robert Laffont.

GILBERT M., 1989, On Social Facts, Princeton, Princeton University Press.

-, 2002, «Belief in Acceptance as Features of Groups», Protosociology, 16, p. 35-69.

HAGENBUCHER SACRIPANTI F., 1992, Santé et rédemption par les génies du Congo, Paris, Publisud. 
HAMAYON R., 2005, «L'anthropologue et la dualité paradoxale du "croire" occidental », Théologiques, |3-I, p. |5-4|.

HAYEK F. A., 1952, Scientism and the Study of Society, Glencoe, The Free Press. HUGUENIN T., 1995, Le 54" ${ }^{\mathrm{e}}$ Paris, Fixot.

JORION P., 1986, «Reprendre à zéro », Revue de l'Homme, 26-97, p. 299-308.

LANG A., 1901, Magic and Religion, Londres, Longmans.

LENCLUD G., 1990, «Vues de l'esprit, art de l'autre. L'ethnologie des croyances en pays de savoir $\gg$, Terrain, 14, p.5-19.

-, 1998, «La culture s'attrape-t-elle?», Communications, 66, p. 165-183.

LÉVI-STRAUSS Cl., 1989 (1950), «Introduction à l'œuvre de M. Mauss » in M. Mauss, Sociologie et Anthropologie, Paris, PUF, p. IX-LII.

-, 1985 (1958), Anthropologie structurale, Paris, Plon.

MACFARLANE A., 1970, Witchcraft in Tudor and Stuart England. A Regional and Comparative Study, Londres, Routledge and Kegan Paul.

MALINOWSKI B., 1992 (1948), Magic, Science and Religion and Other Essays, Prospect Heights, Waveland Press.

MARWICK M. G., 1952, «The Social Context of Cewa Witch Beliefs », Africa, 22, p. $120-135$.

MEIJERS A., 1999, «Believing and Accepting as a Group», in Id. (dir.), Cognition and Will, Tilburg, Tilburg University Press, p. 59-7l.

MERTON R. K., 1968 (1949), Social Theory and Social Structure, New York, The Free Press.

MOORE O. K., 1959, «Divination - a New Perspective», American Anthropologist, LIX, p. 69-74.

NEEDHAM R., 1972, Belief, Language and Experiences, Oxford, Basil Blackwell.

OLSON M., 1978 (1966), Logique de l'action collective, Paris, PUF.

PIETTE A., 2005, La Religion de près, Paris, Métailié. 
POUILLON J., 1979, «Remarques sur le verbe "croire"», in M. Izard et P. Smith (dir.), La Fonction symbolique, Paris, Gallimard, p.43-51.

RADCLIFFE-BROWN A. R., 1972 (1952), Structure et fonction dans la société primitive, Paris, Éditions du Seuil.

RICCEUR P., 1968, «Croyance», Encyclopedia Universalis, Paris, Tome V, p. 17I- 176.

ROSCH E, 1980, «Linguistic Relativity», in P.N. Johnson-Laird et P.C. Wason (dir.), Thinking, Readings in Cognitive Science, Cambridge, Cambridge University Press.

RUSSELL B., 200I (1912), The Problems of Philosophy, Oxford, Oxford University Press.

SANCHEZ P., 2007, La Rationalité des croyances magiques, Genève, Droz.

-, 2009, Les Croyances collectives, Paris, PUF.

SARTRE J.P., 1960, Critique de la raison dialectique, Paris, Gallimard.

SAUVAYRE R., 2012, Croire à l'incroyable, Paris, PUF.

SCHEID J., 1999, Quand faire, c'est croire, Paris, Aubier.

SMITH W. R., 1889, Lectures on the Religion of the Semites, Londres, Adam and Charles Black.

SPERBER D., 1974, Le Symbolisme en général, Paris, Hermann.

-, 1996, La Contagion des idées, Paris, Odile Jacob.

THOMAS K., 1972, Religion and the Decline of Magic, New York, Charles Scribner's Sons.

TUOMELA R., 2000, «Belief versus acceptance», Philosophical Explorations, 2, p. $122-137$.

TURNER V.W., 1957, Schism and Continuity in an African Society, Manchester, University of Manchester.

TYLOR E.B., 1876 (I87I), La Civilisation primitive, Paris, Reinwald Cie éditeurs.

WINCH P., 1986, «Understanding a Primitive Society», in B. R. Wilson (dir.), Rationality, Oxford, Basil Blackwell, p. 78-III. 